\title{
IMPLEMENTACIÓN DE SISTEMAS DE CALIDAD EN LA INDUSTRIA DE LA CONSTRUCCIÓN: HACIA UN MODELO CUALITATIVO DE EVALUACION.
}

\author{
Kevin Luna Villarreal \\ Master en Ciencias con Especialidad en Ingeniería y Administración de la Construcción; \\ Instituto Tecnológico y de Estudios Superiores de Monterrey

\section{Carlos Adrián González Tamez} \\ Master en Ciencias con Especialidad en Ingeniería y Administración de la Construcción; \\ Instituto Tecnológico y de Estudios Superiores de Monterrey. \\ Méjico
}

Remisión Artículo: 10-1-2007

Palabras Claves: sistemas de calidad, ciclo de vida de proyectos de construcción.

\section{INTRODUCCION.}

A lo largo de la historia el hombre ha construido infinidad de edificios e instalaciones que son de naturaleza diversa, pero imperiosos para llevar a cabo el sin número de actividades que son necesarias para la vida diaria. Para llevar a cabo las titánicas labores necesarias para construir esos espacios necesarios, la humanidad se ha dado a la tarea de desarrollar diversas filosofías desde las organizativas hasta las administrativas, entre tantas otras. Las cuales están enfocadas siempre aunque sea de una manera autóctona a facilitar todas las actividades que conllevan la planeación y la ejecución de un proyecto de construcción.

Una de esas tantas filosofías es la calidad, la cual aunque ha sido ampliamente sistematizada y desarrollada desde el siglo XX cuando Demming (1960), aporta sus primeras teorías enfocadas a la producción en serie, y más recientemente cuando Alarcón (1997), ha propuesto el lean construction como una herramienta que está orientada a la industria de la construcción.

Ante el dinámico proceso evolutivo que la calidad ha sufrido en recientes épocas, se ha dado como consecuencia, la generación de un nicho de oportunidad para el desarrollo de especialistas. Este tipo de especialista, es el consultor de calidad y debe de tener un perfil claramente definido, y por supuesto amplia experiencia y conocimiento profundo de la industria.

Así pues el panorama de la calidad dentro de la industria se muestra interesante, pues al contarse con teorías, especialistas y un gran medio donde aplicar y desarrollar nuevas teorías y modelos que permitan estudios y soluciones más eficientes que se traduzcan en lo que significa la calidad, según la define Crosby (1979) como el cumplimiento de las especificaciones. Se podría decir entonces que los aspectos más importantes se encuentran sólidamente cubiertos para garantizar el posicionamiento estratégico y el constante desarrollo de la cultura de la calidad. 
Más sin embargo una pregunta queda en el aire ante tan optimista panorama, para la solución de un problema es necesario forzosamente establecer condiciones generales, y dichas condiciones a efectos prácticos dentro de la industria de la construcción mexicana son propuestas por los especialistas de manera empírica basados en la experiencia a priori que poseen. Dicha experiencia aunque invaluable, no deja de ser hasta cierto punto objeto de discusión entre los especialistas, pues al aplicar criterios discrecionales para el establecimiento de las condiciones iniciales en las etapas tempranas en sus proyectos de consultoría, se deja en tela de duda si dichas apreciaciones son producto de un sesgo de cierta "moda" en la industria, o es una propuesta objetiva y concisa que pretenda resolver los problemas que se han planteado.

Así pues ante esta realidad ha quedado evidenciada la necesidad del desarrollo de una herramienta, que mediante un rápido y sencillo procedimiento permita establecer las condiciones iniciales de una consultoría, mismas que servirán para justificar la elección del sistema de calidad adecuado al caso de estudio.

Para el desarrollo de la herramienta ha sido necesario enfocar el problema desde un punto de vista un tanto global, esto con el objetivo de determinar las actividades genéricas que se presentan en un proyecto así como su naturaleza, mientras por otro lado el análisis de diversos sistemas de calidad

De manera tal que para el desarrollo de la herramienta, la cual pretende establecer las condiciones iniciales que justifiquen la elección de un sistema de calidad determinado, es necesario estudiar entre otras cosas, a la empresa como el ente gestor de los elementos que componen el ciclo de vida de un proyecto de construcción, los sistemas de calidad con cierto grado de aplicabilidad en la industria de la construcción, así como el QFD como técnica que permite analizar qué es lo que se hace y cómo se hace.

\section{LA CONSTITUCION Y EVOLUCION DE LAS EMPRESAS, DE LAS PYME'S HACIA LAS GRANDES EMPRESAS,}

Al hablar de calidad es necesario hablar de manera adyacente de la industria, pues es esta, por las condiciones naturales que posee la que originó el desarrollo de la calidad. Así mismo al hablar de la industria es necesario hablar de los entes que la conforman, es decir, las empresas. Irigoyen (1997), define a una empresa como un ente conformado por individuos que se dan entre sí alguna forma de organización a efectos de coordinar recursos (insumos) con el fin último de lucrar con su transformación o enajenación.

Pero esta definición aunque bastante acertada no deja de ser en todo el contexto una descripción bastante generalizada de una empresa, pues esta además de ser lo anterior es una entidad que tiende a evolucionar, crecer y decrecer conforme fenómenos de origen exógeno, como cambios en el mercado, injieren en su ciclo de vida.

De manera más específica es posible clasificar a las empresas independientemente de la industria o sector al que sirvan según ciertas características generales, esto es en pequeñas y medianas empresas (PyME's) y grandes empresas, esta clasificación se da por el tamaño de su personal y las ventas anuales que generan, en México a través del Diario Oficial de la 
Federación (Diciembre 1993) Tabla $n^{\circ} 1$, se ha especificado una estratificación, de las empresas, con relación a estas variables.

\begin{tabular}{|l|l|l|}
\hline $\begin{array}{l}\text { Tamaño de } \\
\text { Empresa }\end{array}$ & $\begin{array}{l}\text { Número de } \\
\text { Empleados }\end{array}$ & $\begin{array}{l}\text { Ventas Anuales } \\
\text { (Pesos Mexicanos) }\end{array}$ \\
\hline \hline Micro & $0-15$ & $\$ 85,000.00$ \\
Pequeña & $16-100$ & $\$ 850,000.00$ \\
Mediana & $101-250$ & $\$ 1,800,000.00$ \\
Grande & $251-$ más & más de $\$ 1,800,000.00$ \\
\hline
\end{tabular}

Tabla 1: Clasificación de las empresas mexicanas.

Irigoyen (1997), propone además que las PyME's y las grandes empresas cuentan con diferencias bastante polarizadas en seis aspectos fundamentales como son su estructura, sus procedimientos, comportamiento, procesos, gente y contacto.

A raíz de todo lo anterior es necesario resaltar que hay un lugar en donde esta evolución es perfectamente palpable, y este es propiamente dicho la organización de la empresa. Pues una condición natural es, que a medida que una empresa crece, crece el tamaño de su fuerza laboral y por consecuencia la complejidad orgánica de la misma.

El instrumento desarrollado para hacer frente a dicha condición es el organigrama el cual puede ser definido como un modelo gráfico mediante el cual se identifica el sistema de autoridad formal de una organización. Mintzberg (1979) propone que dentro de un modelo de organización se generan distintas acciones genéricas, las cuales a grandes rasgos abarcan la planeación, la organización, la integración, la dirección y el control.

Además de estas acciones Mintzberg propone también que en las organizaciones, se generan distintos mecanismos de coordinación. Entre los más importantes pudiéramos mencionar el ajuste mutuo, la supervisión directa y la estandarización tanto de procesos, habilidades y como consecuencia de resultados.

Esto genera la estructura básica de la organización, en donde se gestan elementos como el cuerpo directivo, los equipos técnico y de soporte, los mandos intermedios y el cuerpo operativo.

Conforme una organización crece y se desarrolla sufre cambios, los cuales se ven reflejados de acuerdo a su complejidad, configuración estructural y rama industrial a la que pertenecen. González (1985), propone en su modelo de evolución típica según la edad de las organizaciones cinco grandes categorías que abarcan a grandes rasgos estos cambios figura $n^{\circ} 1$. 


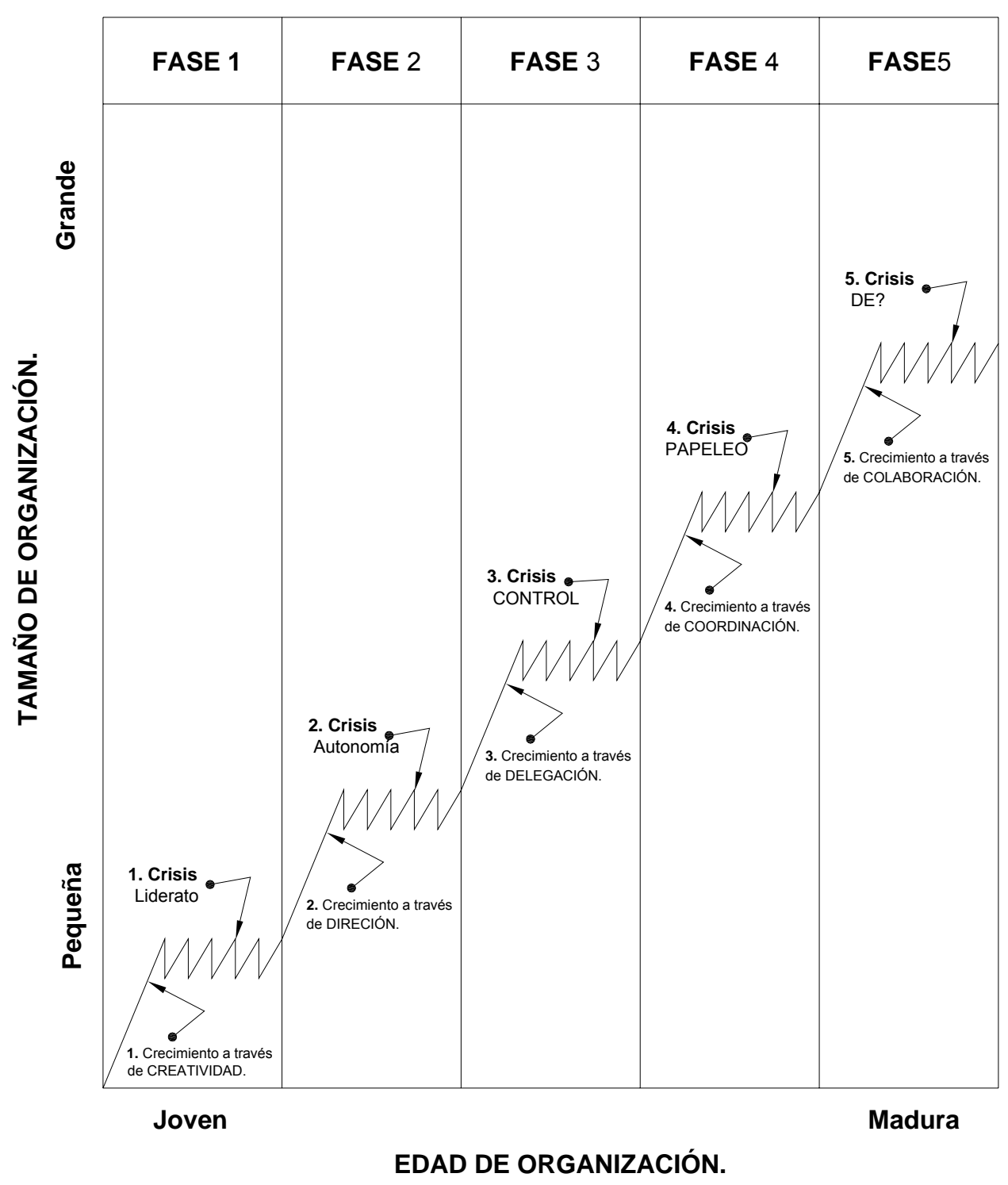

Figura 1: Esquema de crecimiento de una empresa a lo largo del tiempo.

En las organizaciones, el crecimiento es dinámico, y como es posible observar en la figura 1 , cuando una organización crece, se genera una crisis posterior. Así pues por ejemplo, cuando una organización es joven, tiende a crecer con un fuerte apoyo en la creatividad, pero llegará un momento en que esa creatividad generará una crisis de liderato originado por una indefinición de jerarquías, y esto se tiende a resolver a través de la dirección, lo que generaría un departamento de dirección.

Así pues, cuando la empresa sigue su desarrollo natural, llegará un momento en el cual la diversificación de actividades creará una crisis de autonomía, es decir, en la producción de los 
diversos departamentos, ¿Qué tan descentralizados se encuentran de la dirección? La solución llega a través de la delegación de tareas y la entrega de resultados a la dirección. Esto se logra generalmente reformando la organización, para dar cabida a una estructura flexible que permita que permita a las diversas partes la debida interacción entre ellas.

Esto lleva a la generación de otra crisis, que es la del control, para solventar este conflicto es necesario desarrollar un departamento de gerencia de proyectos, que tendría como principales labores el control de los proyectos y por supuesto la coordinación de los mismos.

Como resultado del control y la coordinación, se genera una estructura que genera una crisis de papeleo. El exceso de información y la dificultad para administrar la misma hacen que la estructura labore con cargas de trabajo que merman su productividad de manera substancial. Generalmente una solución es la reestructuración de la organización, para que en adelante se trabaje por proyecto, es decir de manera matricial. Esto genera una colaboración interdepartamental teniendo como consecuencia el trato directo entre especialistas afines al proyecto, simplificando como consecuencia la manera de concebir el trabajo.

\section{EL CICLO DE VIDA DE UN PROYECTO, Y LA APROXIMACION DEL QFD AL MISMO.}

En el apartado anterior analizamos a la empresa como ente, su estructura y su evolución, así pues se ha sentado la base para el análisis del objetivo de una empresa, la cual como ya sabemos es la de transformar los recursos (insumos) para su lucrar con su transformación o enajenación, Irigoyen (1997). A ese objetivo de transformar y lucrar con la transformación de recursos, además de todo lo que se involucra de manera directa o indirecta, podríamos llamarlo proyecto. Dicho de otra manera, un proyecto se compone de un inicio, un final y actividades intermedias, las cuales de acuerdo a su naturaleza e importancia es posible agruparlas en subproductos necesarios para llevar a cabo la realización del producto final, esto entre otros fines, para facilitar su administración, lo cual se conoce también como ciclo de vida.

De manera más específica el ciclo de vida de un proyecto está formado por fases sucesivas que a su vez están compuestas por tareas, que por naturaleza propia son de carácter planificable. El modelo de Morris (2000), propone al ciclo de vida de un proyecto típico de carácter lineal para la programación de los proyectos de construcción figura $n^{\circ} 2$, en el cual de manera indiscutible es posible poder distinguir los elementos que lo conforman tales como las etapas y los entregables (deliverables). 


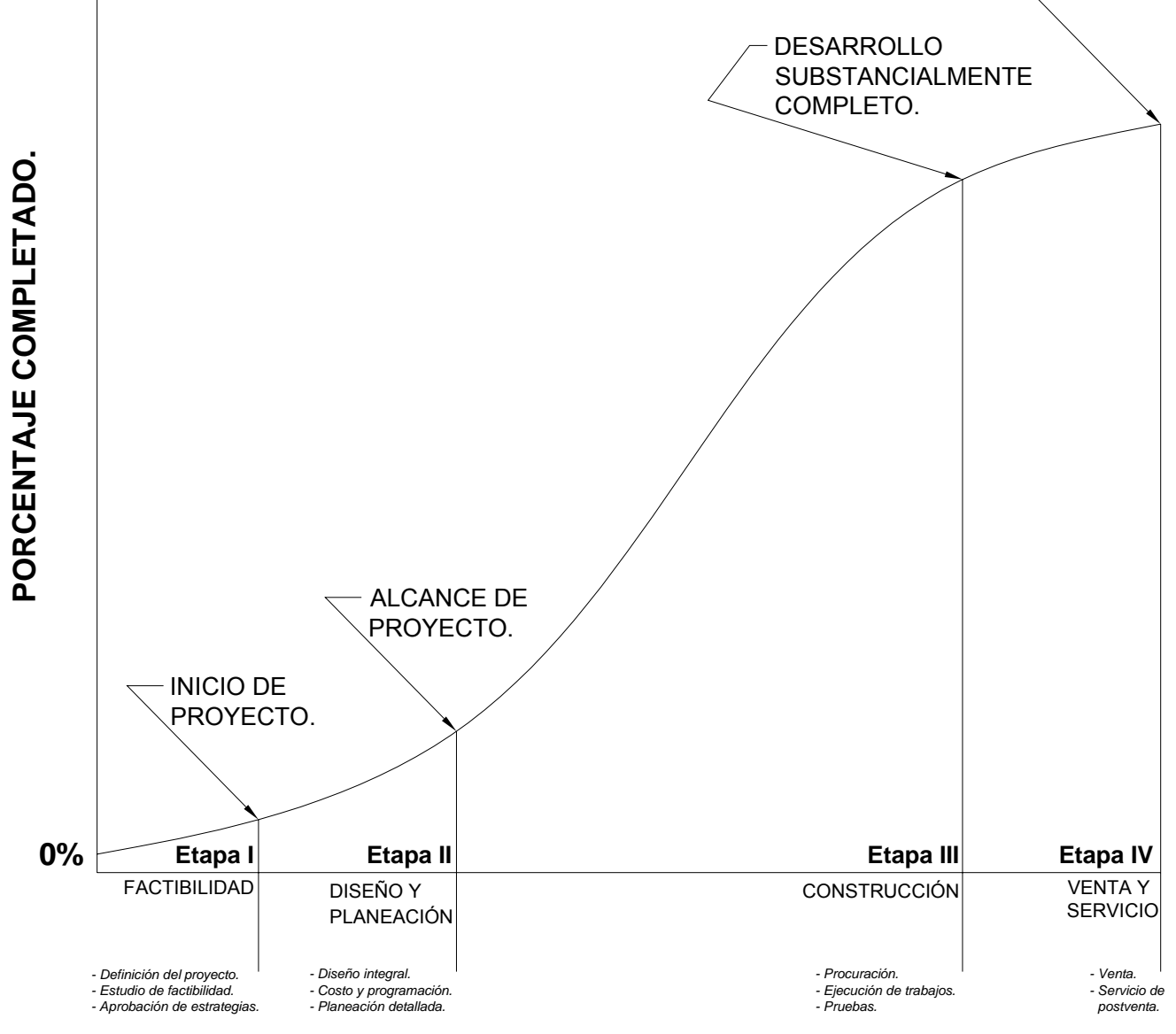

CICLO DE VIDA.

Figura 2 : Modelo de ciclo de vida propuesto por Morris (2000).

Como etapas se podría entender, a grandes rasgos, el conjunto de actividades referidas con el objetivo del proyecto, y éstas se elaboran concentrando tareas, las cuales es posible que compartan un tiempo delimitado en el ciclo de vida del proyecto.

Los entregables (deliverables) como tal son productos que se generan durante las fases. Estos pueden ser concebidos como objetos materiales o inmateriales tabla $n^{\circ} 3$. A su vez los entregables sirven como un estimador que permite realizar una evaluación, y por lo tanto ejercer un control del desempeño real del ciclo de vida del proyecto, cuando este es comparado contra el ciclo de vida proyectado.

\section{EL QFD.}

EI QFD es un método desarrollado para la planeación y desarrollo de estructuras de trabajo que tengan la capacidad de responder a las necesidades del cliente. Esto se logra bajo la 
premisa de evaluar cada producto o servicio en función del impacto en la solvencia de las necesidades del cliente.

EI QFD está constituido por una o varias matrices, llamadas algunas veces "tablas de calidad". La primera de esas matrices es llamada la "Casa de la Calidad" (HOQ). Esta despliega las necesidades del cliente en la derecha y el responsable del área correspondiente el cual está encargado de resolver esa necesidad.

Mas allá de la casa de la calidad, el QFD involucra matrices adicionales, las cuales sirven a futuro como una guía de como el proceso o servicio debe de ser concebido.

La interrelación de matrices es llamada también cadena de matrices. En esta se describen de manera más detallada los productos o servicios que se analizan. Cohen (1995) menciona que existen casos en donde por proceso las cadenas de matrices se han llegado a componer de hasta treinta matrices en donde se detallan todos los procesos tanto administrativos como técnicos que intervienen en esa parte del proyecto, esto da evidencia concreta de lo flexible que es el QFD como herramienta de desarrollo de proyectos.

Como se ha mencionado, el QFD tiene la capacidad de desplegar la "voz del consumidor y sus demandas"1. Estas son localizadas gracias a los múltiples mecanismos de retroalimentación de los cuales se puede servir (investigaciones de mercado, lluvias de ideas, y otras técnicas semejantes) que son trasladados al desarrollo integral del proceso. Para ello, se involucra la traducción de las demandas del cliente en los requerimientos técnicos apropiados según la fase del desarrollo del producto y la producción.

Aguayo (1997) indica que uno de los elementos más importante que el QFD posee para la dirección de proyectos, es la creación de equipos multidisciplinarios de trabajo. Estos equipos deben estar integrados por miembros de todas las áreas que están involucradas en el producto o proceso a diseñar.

El proceso inicia con la identificación de las demandas del cliente ("Voz del Cliente"), las cuales serán, por lo general características cualitativas.

Estas características son importantes para el consumidor, pero muchas veces son difíciles de cuantificar y de llevar a buen término en el proceso de producción. La aplicación del QFD se divide en un proceso de dos partes, figura $n^{\circ} 3$; (1) las matrices uno y dos, que permiten ligar los requerimientos del consumidor con las características de las partes incluidas ${ }^{2}$ y (2) las matrices tres y cuatro, las cuales permiten planear el proceso, así como la planeación de la producción.

\footnotetext{
${ }^{1}$ Por supuesto al hablar de la voz del consumidor y sus demandas entendemos que se habla del cliente, y este puede ser interno o externo, es decir al ser interno, nos referimos a quienes reciben el producto concebido durante las fases de un proyecto, es decir el producto no terminado. Mientras que cuando se habla del cliente externo, generalmente es en referencia al usuario final.

${ }^{2}$ Al hablar del despliegue de las partes incluidas, se hace referencia al involucramiento de recursos necesarios para formalizar el proyecto, tanto humanos, materiales y equipos, los cuales son necesarios para la concepción del proceso de construcción.
} 


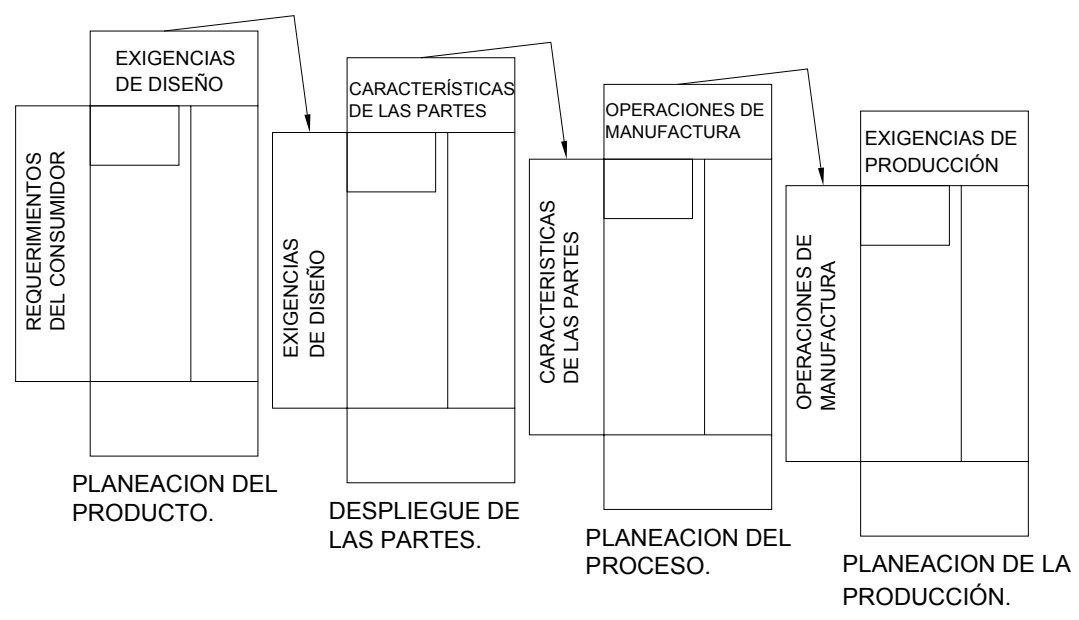

Figura 3: Relación de tablas de calidad acorde al QFD.

\section{CALIDAD A GRANEL.}

Al hablar del QFD y del ciclo de vida de un proyecto, se empieza a esbozar a grandes rasgos la herramienta en sí. En concreto, el QFD permite planear el producto y diseñar el proceso, así como planear el proceso y la ponderación del mismo. Así pues con un dinamismo tal, la herramienta es el resultado de razonar que es lo que se tiene que hacer, pero sobre todo cómo se tiene que hacer, es ahí donde se incorpora la calidad como elemento de ponderación en el ciclo de vida de un proyecto.

La calidad como tal ha sufrido un desarrollo evolutivo consecuencia natural a la condición de progresista de la industria en general, ante este panorama ha sido necesario clasificar y categorizar a los distintos modelos de calidad de acuerdo a su complejidad y aplicabilidad en la industria de la construcción. Específicamente, los modelos que se han estudiado son los siguientes, 5's, Kaizen, ISO 9000, JIT, Lean Construction y Six Sigma.

\section{5'S.}

El concepto de las 5'S se refiere a la creación y mantenimiento de áreas de trabajo más limpias, más organizadas y más seguras, es decir, se trata de imprimirle mayor "calidad de vida" al trabajo. Las 5 'S provienen de los siguientes términos:

- Clasificar, organizar o arreglar apropiadamente: Seiri.

- Ordenar: Seiton.

- Limpieza: Seiso.

- Estandarizar: Seiketsu.

- Disciplina: Shitsuke.

Cuando el entorno de trabajo está desorganizado y sin limpieza se pierde la eficiencia y la moral y la eficiencia en el trabajo se refleja reducida drásticamente. Ante ese panorama el 
objetivo central de las 5 'S es lograr el funcionamiento más eficiente y uniforme de las personas en los centros de trabajo.

Es posible definir las 5'S como:

- Clasificar (seiri). Clasificar consiste en retirar del área o estación de trabajo todos aquellos elementos que no son necesarios para realizar la labor, ya sea en áreas de producción o en áreas administrativas.

- Ordenar (seiton). Consiste en organizar los elementos que han sido clasificados como necesarios de modo que se puedan encontrar con facilidad.

- Limpieza (seiso). Limpieza significa, además de la actividad de limpiar las áreas de trabajo y los equipos, el diseño de aplicaciones que permitan evitar o al menos disminuir la suciedad y hacer más seguros los ambientes de trabajo.

- Estandarizar (seiketsu). El estandarizar pretende mantener el estado de limpieza y organización alcanzado con la aplicación de las primeras 3's.

- Disciplina (shitsuke). La disciplina es el canal entre las 5'S y el mejoramiento continuo. Implica control periódico, visitas sorpresa, autocontrol de los empleados, respeto por sí mismo y por los demás y mejor calidad de vida laboral, además del cumplimiento de las normas y estándares establecidos para conservar el sitio de trabajo en óptimas condiciones.

En síntesis, podríamos decir que el modelo 5's ha sido desarrollado principalmente para su implementación en el campo. Es decir su concepción se basa en el ordenamiento sistemático del sitio de trabajo, lo que trae como consecuencia el aumento de la productividad mediante el orden. Mas sin embargo, el uso de 5's, en las actividades cotidianas realizadas por el staff en una compañía es una consecuencia lógica, esto con ciertas limitaciones. En la construcción como una actividad pluridisciplinaria, el uso de las 5's es especialmente idóneo.

¿Es que acaso no es fundamental tener una disciplina que permita un ambiente de trabajo limpio y ordenado? La respuesta obviamente es sí.

En Europa y más específicamente en las ciudades europeas, la construcción se desarrolla en muchos casos en espacios reducidos, y por igual en lugares públicos y privados, y en ocasiones por esas mismas limitaciones físicas, merman y perjudican la movilidad del ciudadano común.

Es entonces, cuando la idea de racionalizar el uso de cada centímetro cuadrado destinado a un proyecto, tiene sentido para la coexistencia del mismo con la ciudad.

Además el uso del 5's tiene entre otras consecuencias, la disminución de las probabilidades de accidentes ocasionados por el ambiente de trabajo. Pues entre más ordenada y limpia se encuentre una obra, menores son las posibilidades de sufrir un accidente, esto es de especial interés en la industria de la construcción, pues por su naturaleza requiere una movilidad tremenda por parte del personal de campo para realizar sus labores diarias. 


\section{KAIZEN.}

El sistema Kaizen de mejora continua tiene como uno de sus pilares fundamentales el objetivo de eliminar mudas ${ }^{3}$. La atención constante basada en la necesidad de eliminar los factores generadores de improductividades, altos costos, largos ciclos, costosas y largas esperas, desaprovechamiento de recursos, pérdida de clientes, y defectos de calidad, todo lo cual origina la pérdida de participación en el mercado, con caída de la rentabilidad y de los niveles de satisfacción de los consumidores.

\section{¿Qué debe entenderse por desperdicio?}

Según Imai (1998), en cada proceso se agrega valor al producto, y luego se envía al proceso siguiente, por lo tanto los recursos utilizados en cada proceso agregan valor o no lo hacen. Muda por lo tanto implica actividades que no añaden valor económico.

Las siete categorías clásicas

Estas surgen de la clasificación desarrollada por Ohno (1988) y comprende:

- Muda de sobreproducción. La misma es el producto de un exceso de producción, producto entre otros factores de: errores en las previsiones de ventas, producción al máximo de la capacidad para aprovechar las capacidades de producción (mayor utilización de los costos fijos), lograr un óptimo de producción (menor costo total), superar problemas generados por picos de demandas o problemas de producción.

- Muda de inventario. Tiene muchos motivos, y en el se computan tanto los inventarios de insumos, como de repuestos, productos en proceso e inventario de productos terminados.

- Muda de reparaciones / rechazo de productos defectuosos. La necesidad de reacondicionar partes en proceso o productos terminados, como así también reciclar o destruir productos que no reúnen las condiciones óptimas de calidad provocan importantes pérdidas.

- Muda de movimiento. Se hace referencia con ello a todos los desperdicios y despilfarros motivados por los movimientos físicos que el personal realiza en exceso debido, entre otros motivos, a una falta de planificación en materia ergonómica.

- Muda de procesamiento. Desperdicios generados por falta de materia de layout, disposición física de la planta y sus maquinarias, errores en los procedimientos de producción, incluyéndose también las errores en materia de diseño de productos y servicios.

- Muda de espera. Motivado fundamentalmente por: los tiempos de preparación, el tiempo de cola de un elemento para su procesamiento, pérdida de tiempo por labores de reparaciones o mantenimientos, tiempos de espera de ordenes, tiempos de espera

\footnotetext{
${ }^{3}$ Los japoneses utilizan la palabra muda para definir un desperdicio.
} 
de materias primas o insumos. Los mismos se dan también en las labores administrativas.

- Muda de transporte. Despilfarro vinculado a los excesos en el transporte interno, directamente relacionados con los errores en la ubicación de máquinas, y las relaciones sistémicas entre los diversos sectores productivos.

La eliminación de los desperdicios comprende la aplicación de sistemas como el Just in time, mantenimiento productivo total, gestión de calidad total. Así como actividades de grupos pequeños (círculos de calidad, equipos de mejora, equipos para detección, prevención y eliminación de desperdicios), sistemas de sugerencias, y despliegue de políticas.

Kazien como hemos visto, es gestado como una herramienta que tiene como objetivo suprimir en lo posible el desperdicio, y como hemos intuido ese origen es nuevamente en la industria manufacturera. Por tal motivo su adaptación a la construcción es necesario hacerla no de una manera purista. Es decir, como existen diferencias sustanciales en la naturaleza de las actividades de la construcción y la manufactura, esto invita de una manera seductora a reflexionar cómo llevar kaizen a la construcción.

En la construcción, como toda actividad se genera desperdicios, lo cual genera pérdidas. Pero estos desperdicios, ¿Cuándo son costeables y cuando incosteables? Generalmente cuando un presupuesto es realizado, se realiza también el programa de obra, pues de poco sirve conocer el costo de un proyecto si no conocemos la duración del mismo. Pero los presupuestos y las programaciones como es bien sabido se realizan tomando en cuenta desperdicios asumibles tanto de material, mano de obra y equipo. Así mismo el cálculo de la productividad en una actividad se realiza tomando en cuenta que esta no puede ser del $100 \%$. Por lo tanto en la construcción el desperdicio incosteable es por lo menos, aquél que no haya sido estimado en el presupuesto y que no haya sido calculado en la productividad esperada en una actividad.

Esta reflexión invita de manera casi automática otra pregunta ¿Es posible tener más de un desperdicio en una misma actividad o proceso constructivo? Evidentemente sí, en la construcción intervienen una serie de factores tan diversos que disparan las posibilidades de tener más de un tipo de desperdicio. Esta realidad es bien conocida por los residentes y supervisores de obra, pues son estos los que tienen la responsabilidad de lidiar con todos los factores endógenos y exógenos que intervienen en la ejecución de un proyecto. Ante estas condiciones, kaizen, representa una opción asequible para controlar el desperdicio en la construcción, si bien no de una manera purista, si que se puede adaptar con relativa facilidad a la industria de la construcción.

\section{ISO 9000:2000}

Las normas ISO fueron escritas bajo la premisa de que la calidad de un producto no nace de controles eficientes, si no de un proceso productivo y de soportes que operan adecuadamente. La norma ISO 9000 es requerida debido a que garantiza la calidad de un producto mediante la implementación de controles exhaustivos, en donde se asegura que todos los procesos que han participado en su fabricación operan dentro de las características previstas. El punto de 
partida en la estrategia de la calidad es la normalización ${ }^{4}$. Las normas ISO 9000, especifican qué elementos deben integrar el sistema de la calidad de una empresa, y a su vez como deben funcionar en su conjunto estos elementos, para asegurar la calidad de los bienes y de los servicios que se producen. La certificación ISO 9000 puede servir como una forma de diferenciación o "clasificación" de proveedores. Esto es particularmente en áreas de alta tecnología, donde la alta seguridad de los productos es crucial. En otras palabras, si dos proveedores están compitiendo por el mismo contrato, el que tenga un certificado de ISO 9000 puede tener una ventaja competitiva ante algunos compradores.

El certificado ISO 9000 puede también ser un factor competitivo en áreas de productos donde preocupa la seguridad o la confiabilidad. Grandes y pequeñas empresas con negocios a nivel internacional ven en las normas ISO 9000 una vía para conquistar nuevos mercados y mejorar su competitividad. Los requerimientos de las normas ISO pueden variar de sector a sector.

\section{Factores clave para llevar a cabo una buena gestión de la calidad.}

Dentro de la gestión de la Calidad en la empresa existen factores que comprenden la justificación de un buen trabajo, el cual pretende demostrar posteriormente a los auditores el método de trabajo que se ha llevado a cabo, así como sus defectos y sus beneficios. Nava (2003) propone un diagrama figura $n^{\circ} 4$, en que se consideran algunos de los factores más importantes. Estos factores proporcionan una división estructurada del trabajo ${ }^{5}$, partir de la cual se define el método de trabajo y su evaluación, así como la revisión del desempeño y el involucramiento del personal de la empresa y así como sus recursos.

\footnotetext{
${ }^{4} \mathrm{Al}$ mencionar la palabra normalización nos referimos a que los procesos que se evalúan han de tender hacia un comportamiento normal, es decir que estadísticamente han de tender a tener una distribución normal, tratando en lo posible de eliminar datos aberrantes, los cuales producen anormalidad en la distribución.

${ }^{5}$ Esta división se materializa en un equipo, es decir, por una parte un equipo formado por elementos de distintos departamentos involucrados en el proyecto de ISO 9000 y por otra parte los consultores responsables de implementar el sistema de calidad.
} 


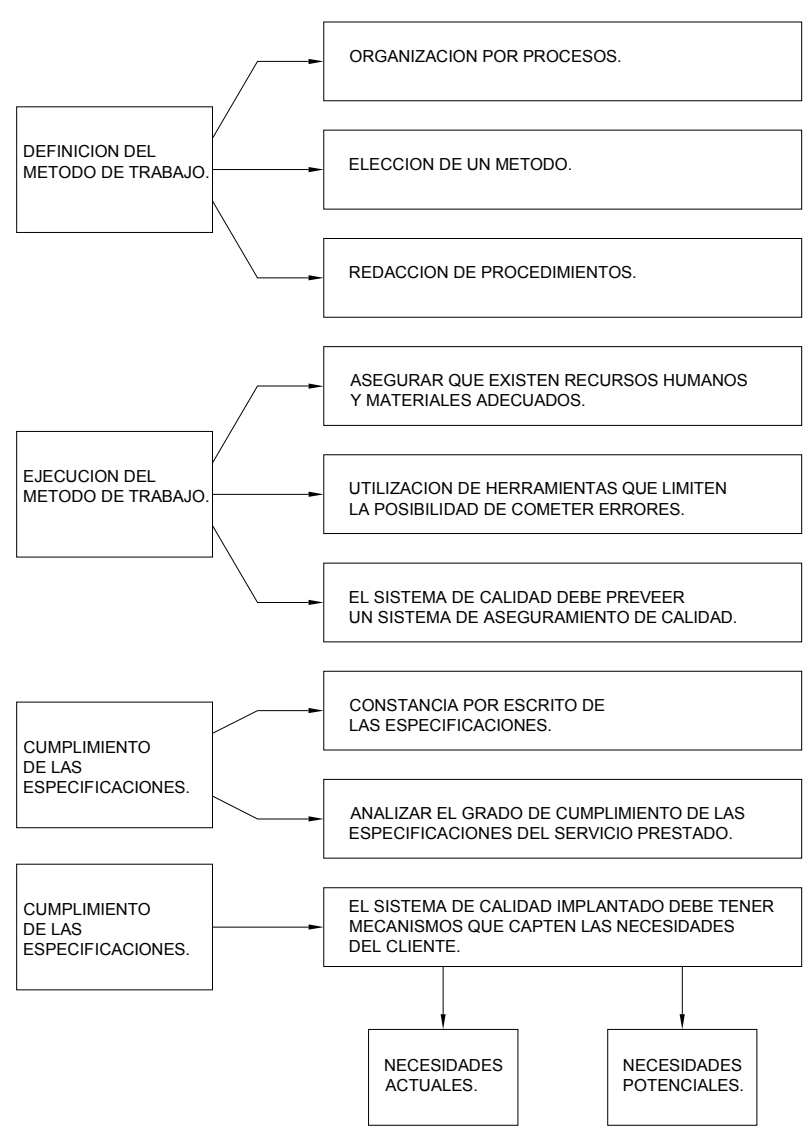

Figura 4: Factores significativos en el proceso de certificación ISO 9000.

Específicamente ISO 9000:2000 plantea los fundamentos de los sistemas de gestión de calidad, así como la terminología que ha de emplearse. El sistema de gestión de calidad de una organización está influenciado por los objetivos de la misma, por sus productos y por sus prácticas específicas. En consecuencia esto varía de una organización a otra. Un sistema de gestión de calidad orienta a la organización a analizar los requisitos del cliente, contar con personal capaz, motivado y dispuesto definir los procesos para la producción. Además de la prestación y el control de los servicios.

Así una vez implantado el sistema de calidad, se procede a realizar las auditorias correspondientes por parte de la empresa certificadora. Aunque los auditores hayan colaborado dentro del proceso de la gestión de calidad, a partir de a aquí comienza una rigurosa evaluación formal, en donde se realizará una pre-auditoria la cual proporcionará un diagnostico claro de errores a corregir. En caso que no existan errores e inconvenientes, los auditores de la empresa certificadora dan su aceptación para llevar a cabo la auditoria final en la cual se concede o no la certificación.

En definitiva ISO 9000 constituye el pilar de la estandarización en la industria. Estandarización que se puede presentar dentro de una empresa tanto en el staff como en producción, o en ambos. Pero para lograr este objetivo un aspecto fundamental es el recurso humano. Pues, para que todo el concepto de la estandarización funcione, es necesario que el recurso humano 
se sumerja en un proceso de capacitación, lo cual evidentemente cuesta a la empresa. Desafortunadamente la industria de la construcción se caracteriza por tener una alta tasa de personal en rotación, sobre todo en el área productiva, y dentro de esta área específicamente en los obreros, lo que hace poco atractiva la idea de capacitar personal que en el corto plazo se terminará marchando al final del proyecto. Esto, evidentemente no se traducirá en un retorno de la inversión sino en una fuga de capital.

Con esta condición, entonces podríamos decir que la capacitación del personal de campo, muchas veces es producto de la experiencia a priori, y no de programas de capacitación fomentados por empresas.

Esto nos lleva a pensar que la aplicación integral del ISO 9000 en una empresa, al menos en México, dada la alta rotación es un objetivo algo ambicioso, más no imposible. Dado que en gran medida como hemos señalado, el grado de complejidad para la implantación del ISO 9000 variará entre otras cosas, por la alta o baja rotación de personal que tenga la empresa. Ante este panorama, la implantación de ISO 9000, pudiera que emanar desde el staff, hacia producción. Esto puede ser, porque desde el staff sería posible además de implantar el modelo de ISO 9000 con relativa facilidad, también sería posible razonar los proyectos con la condicionante de que estos, pudieran diseñarse pensando en procesos de obra que tiendan más a ser de carácter industrial que artesanal, lo cual entre otros beneficios, tendríamos la generación de un ambiente en campo en donde sería más factible empezar sementar las bases de un sistema de calidad.

JIT.

EI JIT es una filosofía gerencial, la cual se enfoca a eliminar fuentes de desperdicio en la producción. Esto se procura mediante la producción de la pieza correcta en el lugar correcto en el momento correcto. El objetivo es el de tratar de eliminar actividades inútiles en cualquier proceso, las cuales agregan un costo sin un valor aditivo al producto ${ }^{6}$. JIT tiene también como objetivo el traducirse en forma de beneficios para la empresa, mediante el retorno de la inversión, además de la reducción en los niveles del inventario. Así mismo, entre otras cosas JIT busca calidad del producto ${ }^{7}$.

EI JIT se aplica sobre todo en procesos de fabricación repetitivos e industrializados. La idea general es establecer procesos del flujo ligando centros del trabajo de modo que haya un flujo de materiales uniforme, equilibrado a través del proceso de producción entero. Para lograr esto, una meta tentativa a alcanzar es la de reducir los tiempos de espera de las colas a cero y otra meta también importante es la de alcanzar el tamaño ideal de producción de un centro de trabajo.

Béranger (1994) menciona los siguientes conceptos como claves para la implementación del JIT.

\footnotetext{
${ }^{6}$ Los fletes y el almacén de materia prima, así como de producto terminado son ejemplos típicos que el JIT considera como actividades inútiles.

${ }^{7}$ La programación de la producción en función de las entregas, así como la reducción de tiempos muertos asociados a la disposición de maquinaria y a los paros de equipo por mantenimiento son también prioridades del JIT.
} 
- Estabilizar y nivelar el MPS (mass production sequence) con una carga de trabajo uniforme en la planta. Crear una carga uniforme en todos los centros de trabajo a través de la planeación de la producción de manera diaria y constante

- Reducir o eliminar los tiempos de disposición. Esto se puede hacer a través de una mejor planeación, del reajuste del proceso, y del reajuste del producto.

- Reducir los tamaños de los lotes (fabricación y compra). La reducción en los tiempos de disposición de materiales, permite la producción económica de lotes más pequeños. Así mismo, la colaboración estrecha con los proveedores es necesaria para alcanzar reducciones en los tamaños de lotes compra, esto se logra con entregas programadas y surtidas continuamente.

- Reducir los tiempos muertos (producción y entrega). Estos pueden ser reducidos moviendo las estaciones de trabajo, agrupándolas, aplicando tecnologías de grupo, o conceptos celulares en la producción. Esto genera una reducción en la longitud de la cola, una mejor coordinación y la cooperación entre los procesos sucesivos.

- Mantenimiento preventivo. Utilizar los tiempos muertos de las máquinas y de los trabajadores para revisar y dar mantenimiento al equipo, esto con el fin de prever interrupciones.

- Fuerza de trabajo flexible. Los trabajadores deben ser entrenados para realizar varios trabajos, para realizar tareas de mantenimiento, así como para realizar inspecciones de calidad.

- Requerir la garantía de calidad del proveedor e implementar un programa de calidad de cero defectos. Los errores que provocan artículos defectuosos deben ser eliminados, puesto que una de las tendencias del JIT, es eliminar el almacén y el costo que este tiene. Por consecuencia se eliminan los espacios intermediarios para el exceso de piezas.

- Fletes de lotes pequeños. Utilizar un sistema de control tal como el sistema Kanban ${ }^{8}$ para transportar piezas entre las estaciones de trabajo en cantidades pequeñas.

En el fondo uno de los aspectos más importantes del JIT es el tiempo. Este es razonado como correctamente como un recurso y es materializado en la programación. Es entonces cuando entendemos la idea del tiempo como variable de primer orden en los proyectos industriales, que el JIT cobra sentido, pues este plantea la organización de toda la producción y el staff alrededor del mismo. Esto tiene como consecuencia espacial, la reorganización de las líneas de producción de una manera tal que puedan ser servidas al momento preciso y con el material requerido. Con esta práctica, como hemos analizado, una consecuencia natural es la eliminación del inventario, y con ello los costes que conlleva tenerlo, pues en términos

\footnotetext{
${ }^{8}$ El Kanban es una tarjeta que se une a un contenedor de transporte. Identifica el número de pieza y la capacidad del contenedor, junto con otra información de relevancia.
} 
monetarios, los costes que se generan por la existencia de un inventario, así como los costes de logística para servir a la línea de producción, son absorbidos por el proveedor.

En la industria de la construcción, aplicar el JIT no es limitarse solo a reducir los inventarios y cargar sus costos a los proveedores, conlleva como hemos visto una serie de factores adyacentes en donde uno de ellos es el tener una relación de empresa mercado, sumamente dinámica, y con factores temporales bastante acotados. Esto como tal es posible tenerlo en la manufactura, que por la naturaleza de los productos es algo fácil de lograr. En la construcción además de que espacialmente la actividad tiene un carácter temporal, es decir, los sitios de construcción se mueven a donde el proyecto ha de realizarse, y no al revés, la relación temporal que la industria guarda con el mercado es algo pasivo en comparación con la industria manufacturera. Lo anterior es posible palparlo por ejemplo en los centros de costo de las empresas, así como en los flujos de efectivo de los proyectos. Por lo anterior es posible entonces decir que la aplicación purista del JIT en la construcción se antoja un tanto complicado, pues en definitiva el JIT esta pensado teniendo en cuenta que se han de cumplir ciertas condiciones con relativa facilidad, las cuales obviamente se cumplen en la manufactura y con grandes esfuerzos y de manera parcial en la construcción.

\section{CONSTRUCCION ESBELTA.}

El concepto de construcción esbelta está formado por varias herramientas, las cuales ayudan a eliminar todas las operaciones que no agregan valor al producto, a un servicio o proceso. Esto produce un aumento en el valor de cada actividad realizada y a su vez elimina lo innecesario. La construcción esbelta ha sido desarrollada a partir de la manufactura esbelta, la cual, nació en Japón y fue concebida por los grandes gurús del Sistema de Producción Toyota: Deming, Ohno, Shingo, Toyoda entre otros.

El sistema de construcción flexible o construcción esbelta ha sido definido como una filosofía de excelencia de construcción, basado en:

- La eliminación planeada de todo tipo de desperdicio.

- El respeto por el trabajador: Kaizen.

- La mejora consistente de Productividad y Calidad.

La construcción esbelta proporciona a las compañías herramientas para sobrevivir en un mercado global, el cual, exige una calidad más alta, una entrega más rápida a precio más bajo y además con la cantidad requerida. Específicamente, Alarcón (1997) considera que la construcción esbelta:

- Reduce la cadena de desperdicios dramáticamente.

- Reduce el inventario y el espacio en el piso de producción.

- Crea sistemas de producción más robustos.

- Crea sistemas de entrega de materiales apropiados.

- Mejora las distribuciones de trabajo para aumentar la flexibilidad. 
La parte fundamental en el proceso de desarrollo de una estrategia esbelta es la que respecta al personal, ya que muchas veces implica cambios radicales en la manera de trabajar.

En perspectiva, la aplicabilidad de este modelo depende en gran medida como en los otros modelos de calidad, del entorno en el cual se quiera implementar. El grado integral de desarrollo industrial, cultural y tecnológico que el modelo requiere, nos lleva a pensar que la aplicabilidad en principio estaría reservada a grandes empresas que por su edad y estructura y posicionamiento en el mercado, pueden asumir sin cambios paradigmáticos a la construcción esbelta como sistema de aseguramiento de calidad. Mas sin embargo, en México la mayoría de las empresas constructoras son PYME's. Pero gracias a técnicas como el benchmarking ${ }^{9}$, y la rotación de personal humano que la industria presenta, este sector del mercado presenta una perspectiva más o menos homogénea en cuestión a su grado de desarrollo integral el cual aunque guardando diferencias, no se encuentra tan rezagado de las grandes empresas. Esto nos induce a reflexionar entonces que en la actualidad, la construcción esbelta es aplicable en cierta medida y con un éxito moderado en un sector considerable de la industria, pero el cambio cultural más que tecnológico que ha de generarse ha de ser estructural, pues este cambio ha de ser efectuado en todos los niveles de la organización.

\section{SIX SIGMA.}

Six Sigma es una forma más inteligente de dirigir un negocio o un departamento. Así pues Six Sigma prioritiza al cliente, usando hechos y datos para estimular mejores resultados.

Eckes (2003), considera que los esfuerzos de Six Sigma deben estar básicamente dirigidos a tres aspectos principales:

- Mejorar la satisfacción del cliente.

- Reducir el tiempo del ciclo.

- Reducir los defectos.

Por lo tanto, es posible definir al Six Sigma como:

- Una medida estadística del nivel de desempeño de un proceso o producto.

- Un objetivo de lograr casi la perfección mediante la mejora del desempeño.

- Un sistema de dirección para lograr un liderazgo duradero en el negocio y un desempeño de primer nivel en un ámbito global.

En la práctica, la medida de sigma es usada para observar que tan bien o mal operan los procesos, y de esta manera darles a todos los procesos una manera común de expresar dicha medida.

\footnotetext{
${ }^{9}$ El benchmarking puede definirse como una técnica que permite comparar, y por lo tanto medir el desempeño de un sistema, con otro similar. El objetivo de esta técnica entre otras el de maximizar el desempeño del sistema y a su vez obtener la mejor relación costo beneficio.
} 


\begin{tabular}{|l|lr|}
\hline NIVEL & DEFECTOS & POR \\
DE & MILLON & DE \\
SIGMA & UNIDADES & \\
\hline \hline 6 & 3.40 & \\
5 & 233.00 & \\
4 & $6,210.00$ & \\
3 & $66,807.00$ & \\
2 & $308,537.00$ & \\
1 & $690,000.00$ & \\
\hline
\end{tabular}

Tabla 2: Relación del nivel de Sigma vs. DPMU.

La meta de Six Sigma, es ayudar a la gente, así como a los procesos, a que aspiren a lograr que los productos y servicios entregados, tengan unas características tales, que se consideren libres de defectos. Si bien Six Sigma reconoce que hay lugar para los defectos pues estos son relativos a los procesos mismos. Un nivel de funcionamiento correcto del 99,9997 por 100 implica un objetivo donde los defectos en muchos procesos y productos son prácticamente inexistentes, tabla $n^{\circ} 3$.

Esto es especialmente ambicioso si se tiene en cuenta que, antes de empezar con una iniciativa de Six Sigma, muchos procesos operan en niveles de 1, 2 y 3 Sigma. Esto ocurre especialmente en áreas de servicio así como administrativas.

De manera paralela, Pyzdek (2003), hace alusión a seis principios que son fundamentales en la filosofía del Six Sigma.

- Enfoque genuino en el cliente. Las mejoras Six Sigma se evalúan por el incremento en los niveles de satisfacción y creación de valor para el cliente.

- Dirección basada en datos y hechos. El proceso Six Sigma se inicia estableciendo cuales son las medidas claves a medir, pasando luego a la recolección de los datos para su posterior análisis.

- Los procesos están donde está la acción. Six Sigma se concentra en el procesos, así pues controlando éstos se lograrán importantes ventajas competitivas para la empresa.

- Dirección pro-activa. Esto significa adoptar hábitos, tales como definir metas ambiciosas y revisarlas frecuentemente. Además es necesario fijar prioridades claras, prevenir problemas y cuestionar la razón por la cual se hacen las cosas, así como la manera en que se hacen.

- Colaboración sin barreras. El objetivo de abolir las barreras físicas y culturales que impiden el trabajo en equipo entre los miembros de la organización, debe de ser una prioridad. Como consecuencia se logra una mejor comunicación, y por tanto, un mejor flujo de información en las labores. 
- Búsqueda de la perfección. Las compañías que buscan aplicar el Six Sigma, tienen en teoría, la meta de lograr una calidad progresivamente más perfecta, y por ende deberían estar dispuestas a aceptar y manejar reveses ocasionales.

\section{¿Como se determina el nivel de Sigma?}

Sigma $(\sigma)$ es un parámetro estadístico de dispersión, que expresa la variabilidad de un conjunto de valores respecto a su valor medio. De modo que cuanto menor sea sigma, menor será el número de defectos. Sigma cuantifica la dispersión de esos valores respecto al valor medio. Por tanto, cuando un cliente define los límites de dispersión, el superior y el inferior con respecto al valor central, tenemos como consecuencia que, cuanto menor sea sigma, menor será el número de valores fuera de especificaciones, y por tanto, el número de defectos será más pequeño.

De tal forma, en la escala de calidad de Six Sigma, se mide el número de sigmas que caben dentro del intervalo definido por los límites de dispersión, de modo que cuanto mayor sea el número de sigmas que caben dentro de los límites de dispersión, menor será el valor de sigma y por tanto, menor el número de defectos.

En concreto, la diferencia entre la Tolerancia Superior (TS) y la Tolerancia Inferior (TI) dividido por la desviación estándar nos da la cantidad (o nivel) de sigmas.

Siempre que la medición esté dentro del intervalo TS-TI se dice que el producto o servicio es conforme o de calidad. En este caso se siguen las ideas de Crosby (1979), quien considera la calidad como sinónimo de cumplimiento de las especificaciones.

En el mundo, las experiencias con el Six Sigma son de naturaleza muy variada, pero en definitiva bastante amplias, resultado de la aplicación en muchas empresas con diversas especializaciones.

En la construcción estas experiencias son contadas, y la literatura es bastante limitada teniendo el caso más concreto en la investigación de Baratta (2005), en donde sugiere que si las empresas cambiaran el enfoque de la aproximación de sus problemas, de una perspectiva orientada a la solución de problemas cuando estos aparecen, a un enfoques en donde se monten ciclos de control causa-efecto, mediante la administración del flujo del valor (VFM), sería posible alcanzar niveles cercanos a los tres sigmas, lo que significa un éxito del más del $90 \%$ en los proyectos.

Pero además, el cambio cultural que se tendría que realizar sería de proporciones mesiánicas. El imaginar que solamente los avances tecnológicos y la capacitación continua son suficientes para asegurar el posicionamiento de Six Sigma en la construcción, es una visión bastante limitada del panorama, pues es sencillamente el adoctrinamiento del recurso humano es el factor clave que permitiría el uso adecuado del Six Sigma.

\section{EL MODELO Y SU EVALUACION.}

\section{ESTABLECIENDO CONDICIONES INICIALES DE ESTUDIO.}

Al estudiar el modelo propuesto por Morris (2000) figura $n^{\circ} 2$, en el que describe el ciclo de vida de un proyecto de construcción. En este modelo, se denota claramente la secuenciación de las 
actividades típicas de un proyecto de construcción. Así pues, estas actividades encierran en su conjunto una serie de subactividades, las cuales a grandes rasgos definen, las consideraciones principales que han de tomarse para llevar a cabo un proyecto. En este modelo se plantean las relaciones existentes entre las etapas iniciales de un proyecto, con la ejecución y servicio del mismo. Así mismo, el modelo plantea en números absolutos, el peso de las diferentes partes ${ }^{10}$. Sin embargo un aspecto fundamental dentro del ciclo de vida del proyecto queda indefinido, esto es, la manera en que se realizan estas actividades, así como las operaciones de manufactura que se realizan en cada fase tabla $n^{\circ} 4$. Por tanto, es necesario complementar este modelo, esto es posible gracias al QFD, que presenta una opción robusta para integrar la calidad al modelo de Morris.

\begin{tabular}{|l|ll|l|}
\hline ENTRADAS & PROCESOS & SALIDAS \\
\hline \hline $\begin{array}{l}\text { Deseo del cliente } \\
\text { (alta dirección) }\end{array}$ & $\begin{array}{l}\text { Definir opciones de } \\
\text { proyecto }\end{array}$ & $\begin{array}{l}\text { Opciones para } \\
\text { Definir opciones de duar }\end{array}$ & \\
Base de datos & $\begin{array}{l}\text { emplazamiento } \\
\text { Definir opciones de } \\
\text { financiamiento }\end{array}$ & \\
& & \\
\hline
\end{tabular}

Tabla 3: Análisis de una tabla de generación de información en una actividad.

\section{DESARROLLO DE UN QFD GENÉRICO PARA EVALUAR LAS VARIABLES DE INTERÉS.}

Una vez determinado el ciclo de vida del producto, es necesario definir con base en los sistemas de calidad propuestos los aspectos a evaluar del ciclo de vida. Como resultado de esto, se obtiene una matriz basada en la metodología del QFD. En esta matriz se evaluarán, los distintos segmentos del ciclo de vida del proyecto, desde el punto de vista de la calidad, cuantificando un resultado en cada uno de estos.

Los sistemas de calidad estudiados han sido acomodados dependiendo de varios factores, los cuales son:

- Complejidad del sistema ${ }^{11}$.

- Costo de implementación del sistema.

- Requerimientos del sistema ${ }^{12}$.

\footnotetext{
${ }^{10}$ Aunque todas las partes de un proyecto son iguales, evidentemente, la etapa de la ejecución del proyecto, es la más crítica, y por tanto la que más peso tiene. Esto es perfectamente apreciable, si medimos un proyecto ya sea con base en el tiempo de ejecución, o en su coste, o con las dos variables de manera simultánea. Técnicas de control de proyectos como el Earned Value, ilustran a la perfección esta tendencia en el comportamiento de los proyectos de construcción a lo largo de su ciclo de vida.

${ }^{11}$ De manera intuitiva es posible afirmar, que hablando de la complejidad de distintos modelos, no es lo mismo implementar un modelo basado en 5'S, que implementar un modelo basado en Six Sigma.
} 


\section{LA PONDERACIÓN.}

El criterio de ponderación propuesto consiste, en la asignación de una calificación a cada aspecto a evaluar. Esta calificación puede oscilar entre $0,1,2$ ó $3^{13}$, según los criterios definidos con anterioridad. Al asignar este tipo de calificación a los criterios, los cuales son de naturaleza cualitativa, se les confiere un peso específico, haciendo posible de esta manera, un estudio cuantitativo de la situación que el modelo pretende reflejar.

Partiendo entonces de lo propuesto con anterioridad, se obtiene una matriz, la cual es capaz de evaluar de manera cuantitativa, el ciclo de vida de un proyecto de construcción desde la perspectiva de distintos modelos de calidad figura $n^{\circ} 5$.

Esta evaluación, permite calificar de manera individual, a cada uno de los departamentos involucrados dentro de un proyecto, así como su desempeño en el mismo.

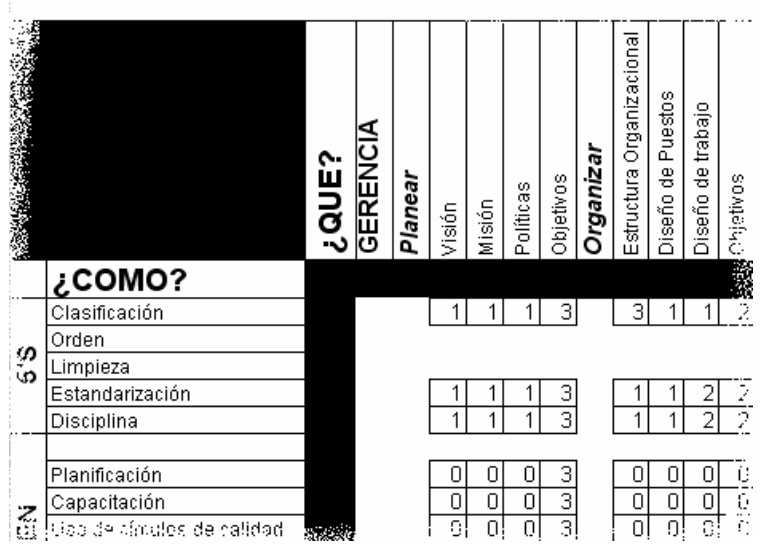

Figura 5: Fragmento de la matriz de ponderación.

\section{EVALUACIÓN DE RESULTADOS.}

La evaluación de los criterios del modelo se pretende que sea en función de lo que se analiza, es decir, el resultado a considerar es el obtenido por la gran media de cada función realizada por los distintos departamentos de una organización, dentro de las distintas etapas del ciclo de vida del proyecto.

El resultado tiene que ser comparado contra una calificación, el cual se propone que sea de 2.25 .

Es necesario tomar en cuenta, que al tomar como criterio la gran media, el rango que compondría cualquier resultado sería de entre 0 a 3 . Entonces, al comparar el resultado con el valor propuesto de 2.25 , si este resulta superior al valor teórico, significaría que por lo menos el

\footnotetext{
${ }^{12}$ Los requerimientos técnicos que los distintos modelos de calidad requieren, son evidentemente diferentes, aunque muchos son complementarios. Esto condiciona en cierta manera una organización jerárquica a priori de los distintos modelos y sistemas de calidad evaluados.

${ }^{13}$ Para efectos de evaluación se han definido estos valores en donde el 0 se otorga cuando existe la total del criterio, se propone 1 cuando los aspectos son definidos deficientemente, 2 cuando están bien definidos y además implementados, y 3 cuando además de estar definidos e implementados existe la disciplina para mantener los criterios, normas y especificaciones, según sea el caso del sistema de calidad evaluado.
} 
75 por 100 de los criterios evaluados reflejan en cierta manera las condiciones necesarias para ser considerados como aceptables ${ }^{14}$. Por tanto, con este resultado, se estaría considerando que el sistema se ha implementado de manera satisfactoria. Esto se refleja en las matrices de evaluación y de resultados, figuras $n^{\circ} 6$ y 7 .

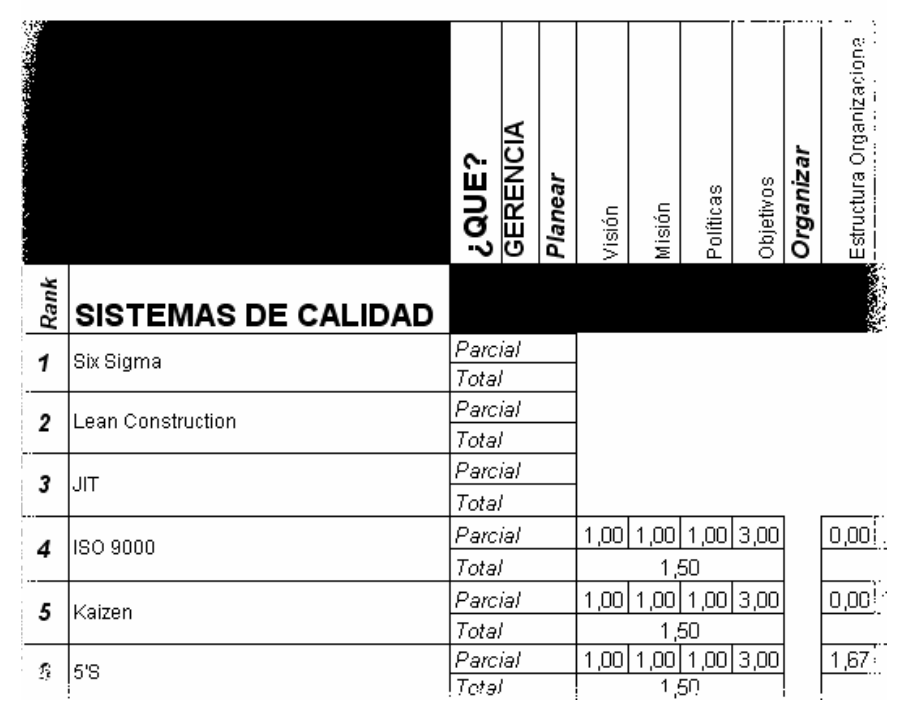

Figura 6: Fragmento de la matriz de evaluación.

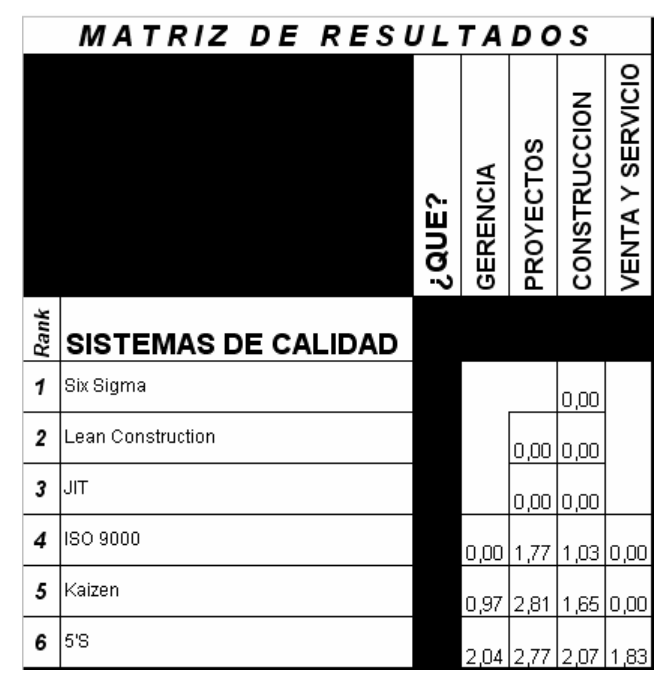

Figura 7: Matriz de resultados.

\footnotetext{
${ }^{14}$ Esto variará de acuerdo al sistema con el que se evalúe cada parte del ciclo de vida del proyecto.
} 


\section{CONCLUSIONES.}

El modelo como tal, representa una alternativa útil y sencilla de aplicar. La implementación en la práctica, se ha llevado a cabo sin mayores complicaciones. Como resultado de esto, se obtiene una perspectiva a priori de la situación global de la empresa. Es posible detectar aquellas áreas de oportunidad, en las cuales es necesario poner especial énfasis a la hora de plantear un modelo de calidad. Con los resultados obtenidos a priori, la posibilidad de justificar la proposición de un modelo de calidad en específico, es materializada. Esto permite a su vez, el desarrollo razonado de un proyecto para la implementación de un modelo de calidad, el cual tiene grandes posibilidades de tener una implementación exitosa.

\section{BIBLIOGRAFIA.}

Oberlender, Garold, 2000, Project management for engineeríng and construction, Boston, McGraw-Hill.

Deming, W. Edwards, 1960, Sample design in business research, USA, Wiley.

Alarcón, Luis, 1997, Lean construction, USA, A.A. Balkema.

Irigoyen, Horacio, 1997, PYMES: su economía y organización, Argentina, Ediciones Macchi.

Mintzberg, Henry, 1979, The structuring of organizations: A synthesis of the research, USA, Prentice-Hall.

González de Hinojosa, Rosa María, 1985, Teoría organizacional: material de apoyo, México, ITESM Campus Monterrey.

Project management institute, 2000, PMBOK guide, USA, Newtown Square.

Cohén, Lou, 1995, Quality function deployment: how to make QFD work foryou, USA, Addison-Wesley

Aguayo Téllez, Humberto, 1997, Modelo del proceso de diseño conceptual: integración de las metodologías QFD, análisis funcional y TRIZ, México, ITESM Campus Monterrey.

Luna Villarreal, Kevin, 2002, Modelo de aseguramiento de calidad basado en el sistema japonés 5 'S aplicado a una empresa de la industria de la construcción, México, ITESM Campus Monterrey.

Imai, Masaaki, 1998, Cómo implementar el kaizen en el sitio de trabajo, Colombia, McGraw-Hill. Ohno, Taiichi, 1988, Workplace Management, USA, Productivity Press.

Nava Carbellido, Víctor Manuel, 2003, ISO 9000:2000: Estrategias para implantar la norma de calidad para la mejora continua, México, Limusa Noriega. 
Béranger, Fierre, 1994, En busca de la excelencia industrial: just-in-time, las nuevas reglas de la producción, México, Limusa.

Eckes, George, 2003, Six Sigma for everyone, USA, John Wiley \& Sons, Inc

Pyzdek, Thomas, 2003, The Six Sigma project planner, USA, Me Graw-Hill.

Crosby, Philip, 1979, Quality is free: the art of making quality certain, USA, McGraw-Hill.

Baratta, Angelo, 2005, NTR03: Achieving Six Sigma level Project Success through the integration of Project Management discipline and Valueflow Management, USA, PMI Global Congress 2005. 